\title{
Elastic Deformations in Strips With Holes Loaded Through Pins
}

\author{
Michael Chi and L. K. Irwin
}

\begin{abstract}
Bickley's solution for the generalized plane stress in a plate loaded through a pin is extended to give the deformation at any point in a plate loaded through one or two pins which are on the same load line. Analytical expressions are derived that are known to apply to a finite strip with a width $\geq 14 a$ and distance between holes $\geq 8 a$, whore $a$ is the radius of the hole. Numerical examples are developed and presented in the form of graphs for typical cases.
\end{abstract}

\section{Nomenclature}

$a=$ radius of the hole

$K_{m}=$ constant coefficients, e.g., $K_{0}=1 / 2, K_{1}=-1 / 12$, $K_{2}=0, K_{3}=1 / 720, K_{4}=0$, etc.

$E=$ Young's modulus of elasticity

$G=$ modulus of rigidity, $\frac{E}{2(1+\mu)}$

$n=$ positive integers from 0 to $\infty$ in an infinite series whose general term is $f(n)$

$n_{0}=$ a positive integer arbitrarily chosen to designate the first term of a partial series

$p=$ maximum intensity of the pressure from the pin on the hole

$P=$ total force exerted by pin on the boundary of hole

$r, \theta=$ polar coordinates

$s=$ partial sum of a series

$u=$ displacement in radial direction

$v=$ displacement in tangential direction

$w_{x}=$ displacement in longitudinal direction

$w_{y}=$ displacement in transverse direction

$\gamma_{r \theta}=$ shearing strain

$\epsilon_{r}=$ strain in radial direction

$\epsilon_{\theta}=$ strain in tangential direction

$\mu=$ Poisson's ratio

$\mu^{\prime}=$ modified Poisson's ratio in a generalized plane stress problem, $\mu /(1+\mu)$

$\sigma_{r}=$ normal stress in radial direction

$\sigma_{\theta}=$ normal stress in tangential direction

$\tau_{\tau \theta}=$ shearing stress

\section{Introduction}

The loads applied to a plate can be transinitted to other plates through joints which are made with fasteners in holes. The loads produce shearing stresses in the fasteners which, in turn, produce compressive or "bearing" forces on the boundaries of the holes. The elastic stresses in the plate produced by the boundary force on a hole, if solved, can be used. directly for the determination of the elastic deformations. These deformations are of primary importance in evaluating the suitability of different combinations of materials and fasteners for aeronautical structures.

This method cannot be applied to a strip of arbitrary dimensions loaded by pins because the general solution for the elastic stresses in this case has not been found. However, the problem of the elastic stresses in an infinite plate loaded by one rigid pin was solved by Bickley [1] ${ }^{1}$ and the problem of a plate with a circular hole at its middle subjected to uniform unidirectional tension was solved by Kirsch [2]. The stresses given by the solutions contained in these papers are combined by superposition to obtain the stresses for the problem considered here. With the aid of these stresses, the deformations of finite strips loaded through one or two holes located on the load line are calculated.

\section{Theory}

Bickley's solution for the general plane stress function $\phi$ for an infinite plate loaded by one solid pin is:

$$
\begin{aligned}
\phi= & a^{2}\left[A_{0} \log _{e} \frac{r}{a}+B_{0} \theta+A_{1}\left\{\frac{r}{a} \theta \sin \theta-\frac{1-\mu}{2} \frac{r}{a}\left(\log _{e} \frac{r}{a}\right) \cos \theta\right\}\right. \\
& +B_{1}\left\{\frac{r}{a} \theta \cos \theta+\frac{1-\mu}{2} \frac{r}{a}\left(\log _{e}^{*} \frac{r}{a}\right) \sin \theta\right\}+C_{1} \frac{a}{r} \cos \theta+D_{1} \frac{a}{r} \sin \theta \\
& \left.+\sum_{2}^{\infty}\left\{a^{m-2} r^{-m+2}\left(A_{m} \cos m \theta+B_{m} \sin m \theta\right)+a^{m} r^{-m}\left(C_{m} \cos m \theta+D_{m} \sin m \theta\right)\right\}\right]
\end{aligned}
$$

where $A_{m}, B_{m}, C_{m}$ and $D_{m}$ are coefficients.

${ }^{1}$ Figures in brackets indicate the literature references at the end of this paper 
The above expression satisfies the biharmonic equation based on compatibility considerations $[3$, p. 54],

$$
\left(\frac{\partial^{2}}{\partial r^{2}}+\frac{1}{r} \frac{\partial}{\partial r}+\frac{1}{r^{2}} \frac{\partial^{2}}{\partial \theta^{2}}\right)\left(\frac{\partial^{2} \phi}{\partial r^{2}}+\frac{1}{r} \frac{\partial \phi}{\partial r}+\frac{1}{r^{2}} \frac{\partial^{2} \phi}{\partial \theta^{2}}\right)=0,
$$

and has single-valued deformations and stresses.

The stresses in polar coordinates in terms of the stress function $\phi$ which satisfy the equations of equilibrium are [3, p. 52]:

$$
\left.\begin{array}{rl}
\sigma_{r} & =\frac{1}{r} \frac{\partial \phi}{\partial r}+\frac{1}{r^{2}} \frac{\partial^{2} \phi}{\partial \theta^{2}} \\
\sigma_{\theta} & =\frac{\partial^{2} \phi}{\partial r^{2}} \\
\tau_{r \theta} & =-\frac{\partial}{\partial r}\left(\frac{1}{r} \frac{\partial \phi}{\partial \theta}\right) .
\end{array}\right\}
$$

The distribution of the forces due to the pin load is customarily assumed to vary as a cosine function over

$$
\begin{aligned}
& \sigma_{r}=-p\left[\frac{1}{\pi}\left(\frac{a}{r}\right)^{2}+\frac{3+\mu^{\prime}}{8} \frac{a}{r} \cos \theta-\frac{1-\mu^{\prime}}{8}\left(\frac{a}{r}\right)^{3} \cos \theta\right. \\
& \left.+\sum_{2,4,6, \ldots}^{\infty} \frac{1}{2 \pi}\left\{\frac{\sin \frac{m+1}{2} \pi \sin \frac{m-1}{2} \pi}{m+1}\right\}\left(\frac{a}{r}\right)^{m} \cos m \theta\left\{2+m\left(1-\frac{a^{2}}{r^{2}}\right)\right\}\right] \\
& \sigma_{\theta}=p\left[\frac{1}{\pi}\left(\frac{a}{r}\right)^{2}+\frac{1-\mu^{\prime}}{8} \frac{a}{r} \cos \theta+\frac{1-\mu^{\prime}}{8}\left(\frac{a}{r}\right)^{3} \cos \theta\right. \\
& \left.\left.+\sum_{2,4,6, \ldots}^{\infty} \frac{1}{2 \pi}\left\{\frac{\sin \frac{m+1}{2} \pi \sin \frac{m-1}{2} \pi}{m+1}+\frac{a}{m-1}\right\}\right)^{m} \cos m \theta\left\{-2+m\left(1-\frac{a^{2}}{r^{2}}\right)\right\}\right] \\
& \begin{aligned}
\tau_{r \theta}=p\left[\frac{\left(1-\mu^{\prime}\right)}{8} \frac{a}{r} \sin \theta-\frac{1-\mu^{\prime}}{8}\left(\frac{a}{r}\right)^{3} \cos \theta\right. & \\
& \left.\left.\quad-\sum_{2,4,6, \ldots}^{\infty} \frac{1}{2 \pi}\left\{\frac{\sin \frac{m+1}{2} \pi \sin \frac{m-1}{2} \pi}{m+1}+\frac{a}{m-1}\right\}\right)^{m} \sin m \theta\left\{m\left(1-\frac{a^{2}}{r^{2}}\right)\right\}\right]
\end{aligned} \\
& \begin{aligned}
\tau_{r \theta}=p\left[\frac{\left(1-\mu^{\prime}\right)}{8} \frac{a}{r} \sin \theta-\frac{1-\mu^{\prime}}{8}\left(\frac{a}{r}\right)^{3} \cos \theta\right. & \\
& \left.\quad-\sum_{2,4,6, \ldots}^{\infty} \frac{1}{2 \pi}\left\{\frac{\sin \frac{m+1}{2} \pi \sin \frac{m-1}{2} \pi}{m+1}\right\}\left(\frac{a}{r}\right)^{m} \sin m \theta\left\{m\left(1-\frac{a^{2}}{r^{2}}\right)\right\}\right]
\end{aligned}
\end{aligned}
$$

Since the stresses at the edge of the hole must be equal to the boundary forces, due to the pin, the coefficients in eq (1) may be determined by substituting eq (1) in eq (2), performing the indicated partial differentiations, and then equating the resulting stresses at the edge of the hole to the forces, eq (3). If the distribution, $f(\theta)$, is assumed acting, the stresses, eq (2), become
Since eq (1) was derived using plane strain theory, the above equations were made applicable for a generalized plane stress solution by replacing Poisson's ratio, $\mu$, with $\mu^{\prime}$ which is equal to $\mu /(1+\mu)$ [5].

In polar coordinates, the stress-strain-deformation relationships are

$$
\left.\begin{array}{l}
\frac{\partial u}{\partial r}=\epsilon_{r}=\frac{1}{E}\left(\sigma_{r}-\mu^{\prime} \sigma_{\theta}\right) \\
\frac{\partial v}{\partial \theta}+u=r \epsilon_{\theta}=\frac{r}{E}\left(\sigma_{\theta}-\mu^{\prime} \sigma_{r}\right) \\
\frac{1}{r} \frac{\partial u}{\partial \theta}+\frac{\partial v}{\partial r}-\frac{v}{r}=\gamma_{r \theta}=\frac{1}{G} \tau_{r \theta^{\circ}}
\end{array}\right\}
$$


Substituting the first and second expressions of (4) into the first part of eq (5) and integrating,

$$
\left.u=\int \epsilon_{r} d r\right]_{\theta=\text { const }}=\frac{1}{E} \int\left(\sigma_{r}-\mu^{\prime} \sigma_{\theta}\right) d r
$$

similarly,

$$
v=\frac{1}{E} \int r\left(\sigma_{\theta}-\mu^{\prime} \sigma_{r}\right) d \theta-\frac{1}{E} \int\left(\sigma_{r}-\mu^{\prime} \sigma_{\theta}\right) d r
$$

By substituting (6) into the last of eq (5), it can be shown that the constants of integration are rigid body displacements [3, p. 189] and therefore may be set equal to zero for the purposes of this investigation.

Thus far, only the infinite plate with the load applied through a pin at the middle and supported at infinity [6] as shown in figure 1(a) has been considered. The complete solution of the problem, figure 1(c), is obtained by superposing the solutions of two subproblems, figures 1 (a) and 1 (b). The problem of a plate with a circular hole at its middle and subject to a uniform unidirectional tension, figure 1(b), is the well known Kirsch's problem which can be found in standard texts [3, p. 195].
$1 / 2 P$

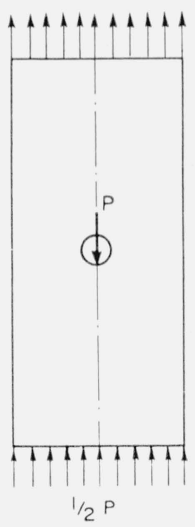

(a)
$1 / 2 P$

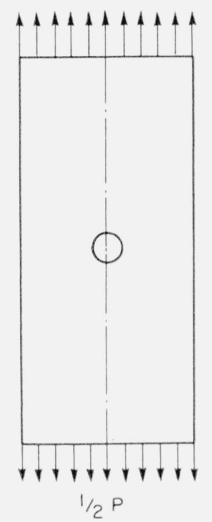

(b)

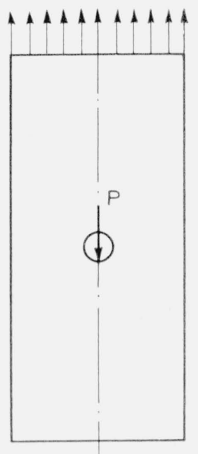

(c)

FIGURE 1. Superposition of subproblems (a) and (b) to solve problem (c).

Sketches represent infinite plates.

Combining these two solutions, the equations for radial and tangential displacements are, respectively,

$$
\begin{aligned}
& \varepsilon=\frac{p a}{E}\left[\frac{1+\mu^{\prime}}{\pi} \frac{a}{r}+\frac{3+2 \mu^{\prime}-\left(\mu^{\prime}\right)^{2}}{8}\left(\log _{e} \frac{a}{r}\right) \cos \theta+\frac{1-\left(\mu^{\prime}\right)^{2}}{16} \frac{a^{2}}{r^{2}} \cos \theta\right. \\
& \left.+\sum_{2,4,6, \ldots}^{\infty} \frac{1}{\pi}\left(\frac{a}{r}\right)^{m-1}\left\{\frac{\sin \frac{m-1}{2} \pi \sin \frac{m+1}{2} \pi}{m-1}+\frac{\frac{m}{2}+1+\left(\frac{m}{2}-1\right) \mu^{\prime}}{m+1}\right\} \frac{\frac{m}{2}\left(1+\mu^{\prime}\right)}{m+1} \frac{a^{2}}{r^{2}}\right\} \cos m \theta \\
& \left.+\frac{\pi\left(1-\mu^{\prime}\right)}{128} \frac{r}{a}+\frac{\pi\left(1+\mu^{\prime}\right)}{128} \frac{a}{r}+\left\{\frac{\pi}{32} \frac{a}{r}+\frac{\pi\left(1+\mu^{\prime}\right)}{128} \frac{r}{a}-\frac{\pi}{128}\left(1+\mu^{\prime}\right) \frac{a^{3}}{r^{3}}\right\} \cos 2 \theta\right]
\end{aligned}
$$$$
v=\frac{p a}{E}\left[\frac{1+2 \mu^{\prime}+\left(\mu^{\prime}\right)^{2}}{8} \sin \theta-\frac{3+2 \mu^{\prime}-\left(\mu^{\prime}\right)^{2}}{8}\left(\log _{e} \frac{a}{r}\right) \sin \theta+\frac{1-\left(\mu^{\prime}\right)^{2}}{16} \frac{a^{2}}{r^{2}} \sin \theta\right.
$$

$$
\begin{aligned}
+\sum_{2,4,6, \ldots \pi}^{\infty} \frac{1}{\pi}\left(\frac{a}{r}\right)^{m-1}\left\{\frac{\sin \frac{m-1}{2} \pi}{m-1}+\frac{\sin \frac{m+1}{2} \pi}{m+1}\right\}\left\{\frac{-2+\frac{m}{2}\left(1+\mu^{\prime}\right)}{m-1}-\frac{\frac{m}{2}\left(1+\mu^{\prime}\right)}{m+1} \frac{a^{2}}{r^{2}}\right\} \sin m \theta \\
\left.-\left\{\frac{\pi}{64}\left(1-\mu^{\prime}\right) \frac{a}{r}+\frac{\pi}{128} \frac{r}{a}\left(1+\mu^{\prime}\right)+\frac{\pi}{128}\left(1+\mu^{\prime}\right) \frac{a^{3}}{r^{3}}\right\} \sin 2 \theta\right] .
\end{aligned}
$$

$\mu^{\prime}$ is taken as 0.25 for the purpose of this investigation.

The displacements in Cartesian coordinates can be evaluated from the radial and tangential displacements in polar cordinates by the following relationship [4, p. 18]:

$$
\left.\begin{array}{l}
w_{x}=u \cos \theta-v \sin \theta \\
w_{y}=u \sin \theta+v \cos \theta
\end{array}\right\}
$$

Therefore, the displacements at any point in the infinite plate loaded through a pin can be found by substituting eq (7) into eq (8).

The displacements thus obtained are meaningless by themselves because they include an arbitrary amount of rigid body displacements. The displacements of interest are the relative displacements between the points in the plate and a chosen reference point. The point, $r=a, \theta=0$ is chosen as the 
reference point. The displacement of any point is taken to be the difference between the displacement of that point as computed by eq (8) and the displacement of the reference point.

The rate of convergence of the series in the equations for the displacements, eq (7), varies with the distance of the point from the hole. The first five terms were sufficient to give an accuracy within one-half percent for the displacements at all points. The relative displacements are the differences between large numbers of the same order of magnitude. To insure sufficient accuracy in the final results, the displacements in eq (7) were therefore carried to nine significant figures. This involved, for example, the inclusion of 6 terms of the series for the points a distance 3 times the radius of the hole, $3 a$, from the edge of the hole and 16 terms for points a distance $0.5 a$ from the edge of the hole. For comparable accuracy at the edge of the hole, about 100 terms of the series would be required. To reduce the computational task, the following Euler-Maclaurin Formula for computing the partial sum of the series was employed.

$\left.s=\sum_{0}^{n_{0}-1} f(n)+\int_{n_{0}}^{n_{0}+1} f(n) d n+\sum_{m=0}^{\infty} K_{m} 2^{m} \frac{d^{m}}{d n^{m}} f(n)\right]_{n_{0}+1}^{n_{0}}$.

In this case, $f(n)=\frac{2}{\pi} \frac{4 n+1-\mu^{\prime}}{\left(4 n^{2}-1\right)^{2}}$.

\section{Finite Strip With One Hole}

In a plate of finite width, a hole close to an edge of the plate may have considerable effect on the stress distribution in the plate. The case of a plate of finite width (strip) with a circular hole on the axis of a symmetry was solved by a method of successive approximations [7]. The method was later extended to the case in which the hole is loaded through a pin [8]. However, the strain expressions obtained by this method were not suitable for integrating to obtain the displacements.

For strips whose width is large compared with the diameter of the hole, ratio of diameter of hole to width of strip less than 0.15 , the effects of the edges of the strip on the stresses around the hole can be neglected [9]. On this basis, the displacements of an infinite plate due to a load applied through a pin are assumed to be applicable to a finite strip without serious error. Figure 2 shows the relative displaces ments along the longitudinal centerline and the edgefor a strip with one pin. The diameter to width ratio is equal to 0.125 .

\section{Finite Strip With Two Holes}

The stresses around a hole are influenced by the presence of adjacent holes. The method of successive approximations is again applicable to this case [10] but becomes much more complicated for the case of two or more holes with boundaries loaded through pins.

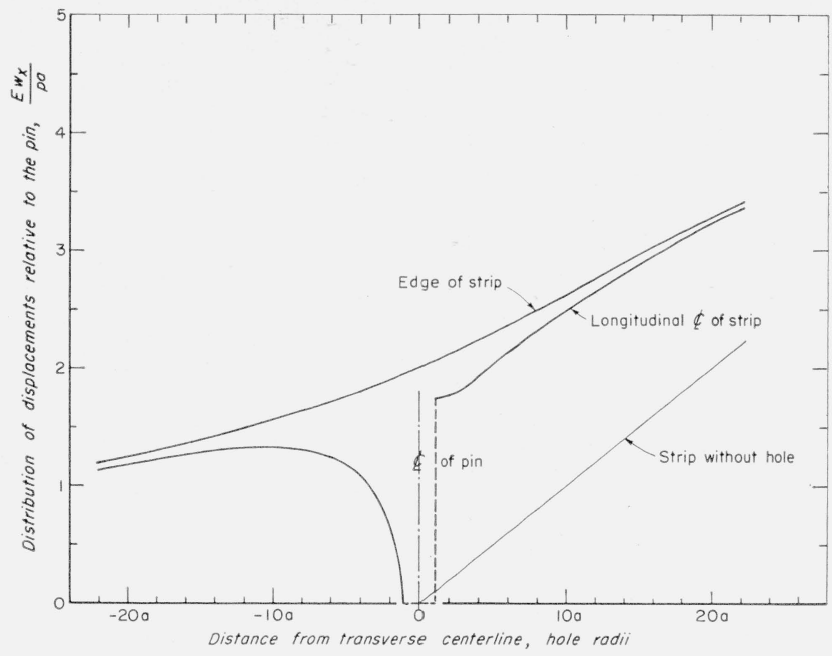

FIGURE 2. Relative displacements along the longitudinal centerline and the edges for a strip with one pin.

As previously mentioned, the effect of a hole in a plate is a local phenomenon, and the effects of adjacent holes on each other can be neglected [9] if they are sufficiently far apart; that is, if the center to center distance of the holes is four or more times the diameter of the larger hole. Thus for a spacing exceeding four hole diameters, the displacement of a point in a plate containing two holes loaded through pins can be taken equal to the sum of the displacements due to each hole taken separately.

The relative displacements along the longitudinal centerline and along the edges of a strip with two equidiameter holes located on the longitudinal centerline and loaded through pins are shown in figure 3. The holes are four diameters apart and the ratio of the diameter of the hole to the width of the strip equals 0.125 .

\section{Concluding Remarks}

The analytical expressions and the computational methods presented here are applicable for determining the elastic deformations of finite strips loaded by pins when the effects of edges and interference between adjacent holes are negligible. Such strips have widths $\geq 14 a$ and distances between holes $\geq 8 a$. The examples for which deformations are shown in figures 2 and 3 correspond directly to single-shear test specimens widely used to evaluate the performance of joints. Further studies should be made to extend the range of these results.

This investigation was part of a project conducted under the sponsorship of the Bureau of Aeronautics. The authors wish to thank this agency for its support of this work. 


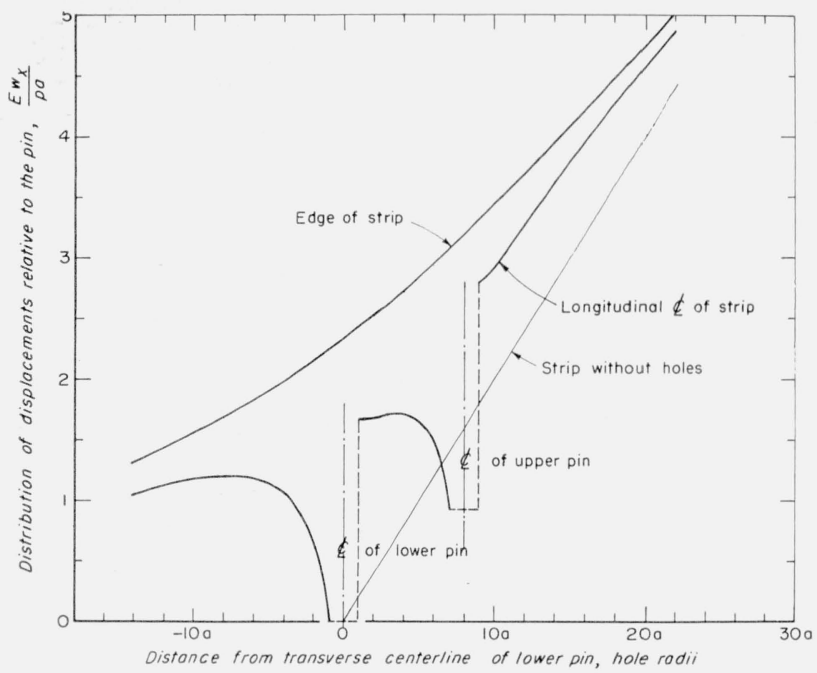

Figure 3. Relative displacements along the longitudinal centerline and the edges for a strip with two pins.

\section{References}

[1] W. G. Bickley, Phil. Trans. Roy. Soc. (London) [A 22\%, 383 (1928).

[2] G. Kirsch, Z. Ver, deut. Ing, 42, 797 (1898)

[3] C. T. Wang, Applied elasticity (McGraw-Hill Book Co., New York, N.Y., 1953).

[4] S. Timoshenko and J. N. Goodier, Theory of elasticity, $2 \mathrm{~d}$ ed. (McGraw-Hill Book Co., New York, N.Y., 1951).

[5] A. E. H. Love, A treatise on the mathematical theory of elasticity, 4th ed.. pp. 103, 138, and 208 (Dover Publications, New York, N.Y., 1944).
[6] R. C. J. Howland, Proc. Roy. Soc. (London) [A] 124, 101 (1929).

[7] R. C. J. Howland, Phil. Trans. Roy. Soc. (London) [A] 229, 49 (1930).

[8] R. C. J. Howland, Proceedings of the Third International Congress of Applied Mechanics, vol. II, 74 (1930).

[9] A. Atsumi, J. Appl. Mechanics 23, 555 (1956).

[10] R. C. J. Howland, Proc. Roy. Soc. (London) [A] 148, 471 (1935).

Washington, November 13, 1958. 